\title{
红外硫系光学玻璃条纹度测量及表征方法研究
}

\author{
胡向平 ${ }^{1}$, 徐光以 ${ }^{1}$, 麦绿波 ${ }^{2}$, 沈义梅 ${ }^{1}$, 杨 静 $^{3}$, 陈 振 $^{1}$, 李建新 ${ }^{1}$ \\ (1. 湖北新华光信息材料有限公司, 湖北 襄阳 441057; 2. 中国兵器工业标准化研究所, 北京 100089 ;
}

3. 昆明物理研究所, 云南 昆明 650223)

\begin{abstract}
摘要: 红外硫系光学玻璃属于不透可见光的玻璃材料, 采用 GB/T 7962 不能进行条纹度测试。本文针 对上述材料中条纹度难以检测与表征的问题, 提出了基于平行光投影法的测试方法, 开发了相应测试 设备, 并对制备的硫系玻璃样品进行了条纹度、灰度及调制传递函数（MTF）测试，研究了灰度和调 制传递函数（MTF）的对应关系，提出了用灰度表征条纹严重程度的方法。结果表明：平行光投影法 在近红外波段成像能清晰地检测到样品内部条纹缺陷和分布; 中红外调制传递函数(MTF) 可定性表 征样品中条纹对成像质量的影响; 条纹度可定量表征样品内部条纹状况, 反映对成像质量的影响。为 后续该材料的内部质量判定奠定了技术基础。
\end{abstract}

关键词: 红外硫系光学玻璃; 不透可见光; 条纹度; 投影法; 灰度表征

中图分类号：TN213 文献标志码：A 文章编号：1001-8891(2020)08-0769-06

\section{Measurement and Characterization Method of Striae for Infrared Chalcogenide Optical Glass}

\author{
HU Xiangping ${ }^{1}$, XU Guangyi ${ }^{1}$, MAI Lvbo ${ }^{2}$, SHEN Yimei ${ }^{1}$, YANG Jing ${ }^{3}$, CHEN Zhen ${ }^{1}$, LI Jianxin ${ }^{1}$ \\ (1. Hubei New Huaguang Information Materials Co., Ltd., Xiangyang 441057, China;
}

2. China Ordnance Industry Standardization Institute, Beijing 100089, China; 3. Kunming Institute of Physics, Kunming 650223, China)

\begin{abstract}
Infrared chalcogenide optical glass is a material that is opaque to visible light. The method outlined in GB/T 7962 cannot be used to test its striae. Herein, test methods based on parallel light projection are presented to facilitate striae detection and characterization. The corresponding test equipment (chalcogenide glass samples) are developed and tested for striae and grayscale and modulation transfer function (MTF); further, the relationship between grayscale and MTF is investigated. Subsequently, the characterization method of striae severity with grayscale is proposed. The results show that the defects and distribution of the inner striae can be clearly detected after imaging in the near infrared band by parallel light projection. The mid-infrared MTF can qualitatively characterize the effect of striae on imaging quality. The striae can be used to quantitatively characterize the internal striae condition of the sample and reflect the effect on imaging quality. This paper provides a reference for the subsequent determination of the internal quality of infrared chalcogenide optical glass.
\end{abstract}

Key words: infrared chalcogenide optical glass, opaque to visible light, striae, projection method, gray scale characterization

\section{0 引言}

近年来, 随着非制冷型探测器制造技术的突破性 进展, 红外光学系统在军用和民用领域有了广泛的应 用。而对于光学设计者来说, 红外硫系光学玻璃在 $60^{\circ} \mathrm{C} \sim 80^{\circ} \mathrm{C}$ 以上 ${ }^{[1]}$ 比 $\mathrm{Ge}$ 单晶的透过性能更好, 热膨
胀系数 (coefficient of thermal expansion, CTE) 和折射 率温度系数 $(\mathrm{d} n / \mathrm{d} T)$ 的配合使用减少了温度函数焦移, 且精密玻璃模造成型（precision glass molding, PGM) 是生产高产量、高性能红外镜头最合算的方法 ${ }^{[2]}$ 。因 此该材料在军用肩扛枪瞄、车载夜视、安防监控和电 力故障检测等领域具有广阔的应用前景 ${ }^{[3-4]}$ 。材料的内 
部品质决定了光学系统的成像清晰程度, 特别是光学 玻璃内部条纹是影响光学成像质量的最重要因素。而 目前用摇摆炉制造红外光学硫系玻璃的方法决定了 产品条纹的质量一致性与晶体材料相比控制困难。因 此对于这类材料条纹度的测量就显得尤为重要, 而该 类产品的透射波段为 $0.78 \sim 20 \mu \mathrm{m}$, 由于不透可见光， 不能采用无色光学玻璃的条纹度测试方法 ${ }^{[5-6]}$ 进行条 纹检测 $[7-8]$ 。但条纹度又是表征产品内在质量的关键技 术指标, 直接影响到红外成像系统图像清晰度。而国 外尚未报道红外硫系光学玻璃条纹度的测试方法或 评价标准, 因此, 本文提出了不透可见光红外硫系玻 璃的测试方法, 并提出了条纹处灰度新的表征方法, 解决了不透可见光玻璃的条纹测试及定量表征问题, 为红外硫系光学玻璃的品质提升及市场应用奠定了 技术基础。

\section{1 测试原理研究}

本文提出了基于平行光投影法, 用均匀的准直近 红外光照射被测试样, 通过条纹处的光线由于折射率 不同会发生偏折, 会聚镜头组完成了标准红外眼的设 计, 利用红外成像系统采集并显示该试样的条纹影像 严重程度, 并对条纹区域的灰度进行判读。红外光学 硫系玻璃条纹测试原理见图 1。

红外光源位于准直光学系统的焦点位置, 红外光 源发射的红外光, 经过准直光学系统后变成平行光, 平行光通过内部有条纹的样品时, 由于样品内存在条 纹, 条纹处材料的折射率与周围材料的折射率不同, 因而红外光源发出的光经过被测样品后光线会发生 偏折, 并形成投影像, 该投影像经聚焦光学系统后投 射在红外图像传感器的靶面上, 通过计算机处理系统 采集红外图像传感器靶面上的图像, 并显示在电脑显 示屏上。根据显示器的图像进行分析计算。

\section{2 实验过程}

\section{$2.1 \mathrm{As}_{40} \mathrm{Se}_{60}$ 玻璃样品的制备}

将纯度为 $99.99 \%$ 以上的单质 As 和单质 Se 按照 原子百分比 $\mathrm{As}: \mathrm{Se}=40: 60$ 计算所需原材料, 并在真 空配料箱中称量所需原料装入安埕瓶中, 然后将安 瓿瓶取出, 与真空设备连接, 抽真空, 使其真空度 达到 $10^{-3} \mathrm{~Pa}$, 将安瓿瓶焊接密封, 然后放在摇摆炉中, 温度升至 $900^{\circ} \mathrm{C}$, 保温 $10 \mathrm{~h}$, 并按照一定频率摇摆, 然后将温度降到 $600^{\circ} \mathrm{C}$, 将安鶕瓶取出降温至 $300^{\circ} \mathrm{C}$, 放入退火炉中, 按照 $-4^{\circ} \mathrm{C} / \mathrm{h}$ 的降温速率降至室温取 出, 将安瓿瓶打破取出玻璃。

\section{$2.2 \mathrm{As}_{40} \mathrm{Se}_{60}$ 玻璃样品条纹严重程度的测试}

2.2.1 红外光学玻璃条纹度测试

利用上述测试原理开发了红外材料条纹专用检 测仪器, 见图 2。

本测试仪由近红外光源、准直光学系统、聚焦 光学系统、红外图像传感器、计算机处理系统以及 显示器组成。打开线路开关, 调节电流旋钮使电流 升至 $133 \mathrm{~A} ， 10 \mathrm{~min}$ 后接通计算机。调节支架，使其 接近镜头中心位置的零位, 将样品放到支架上。启动 程序 glass.exe, 调节移动接收镜头, 使显示屏上的画 面边缘不发生变暗（变亮）情况。通过调焦装置和 glass.exe 程序辅助, 在显示屏上可以得到清晰的条纹 图像, 根据此图像判断样品的条纹状况。

设备技术要求:

(1)样品测量尺寸: $\leqslant \phi 150 \mathrm{~mm}$ 。(2)分辨率: $0.05 \mathrm{~mm}$ 宽线状条纹和 $0.1 \mathrm{~mm}$ 点状缺陷。(3)测量精度: $\leqslant 0.1 \mathrm{~mm}$ 。 (4)环境要求: 温度: $22^{\circ} \mathrm{C} \pm 5^{\circ} \mathrm{C}$ 。(5)样品要求: 两通 光面平行并抛光, 平行度 $\leqslant 0.1 \mathrm{~mm}$, 表面光洁度: $60 / 40$ 。

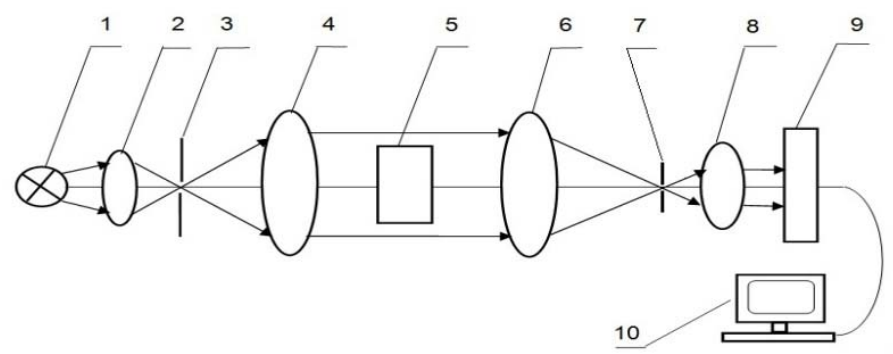

1. 光源; 2. 聚光镜; 3. 光源光阑; 4. 准直透镜; 5. 试样; 6. 会聚透镜 $1 ; 7$. 孔径光阑; 8. 会聚透镜 2; 9. 图像传感器; 10. 计算机图像采集、处理与显示系统

1. Light source; 2. Collecting lens; 3. Light aperture; 4. Collimating lens; 5. Sample; 6. Convergent lens 1; 7. Aperture diaphragm; 8. Convergent lens 2; 9. Imaging sansor; 10. Image collecting, processing and display system of the computer

图 1 红外光学硫系玻璃条纹测试原理图

Fig.1 Principle diagram of striae measurement for infrared chalcogenide optical glass 


\subsection{2 $\mathrm{As}_{40} \mathrm{Se}_{60}$ 玻璃样品传递函数的测试}

采用 TRIOPTICS-MTF-LAB 设备在温度 $22^{\circ} \mathrm{C}$ 下 对平行度 $\leqslant 0.1 \mathrm{~mm}$, 表面光洁度优于 $60 / 40$ 的样品进 行测试, 测试波长 $4000 \mathrm{~nm}$ 。用 $f / 2$ 的中波红外镜进行 对比评价。测量精度 \pm 0.02 (轴上) 、 \pm 0.03 (轴外)。

\subsection{3 $\mathrm{As}_{40} \mathrm{Se}_{60}$ 玻璃样品灰度值测试}

利用本原理制造的红外条纹仪测试设备（如图 2）。打开电源, 预热 $10 \mathrm{~min}$ 以上, 打开 MV-Demo 测试软件, 将样品放置在样品架上, 样品中的条纹状 况就会显示在计算机的屏幕上, 打开 Cam-config 软 件, 选定样品区域, 输入样品尺寸, 点击测试区域没 有条纹区域的灰度值, 设定为标准灰度, 然后点击有 条纹区域负的各点, 即可显示各点的相对灰度值; 在 显示器上选择试样边框, 输入试样尺寸; 设置灰度范 围, 即可直接读出条纹面积占试样的百分比和灰度的 标准偏差值。然后用灰度的标准偏差和条纹所占面积 之积来表示其条纹度。

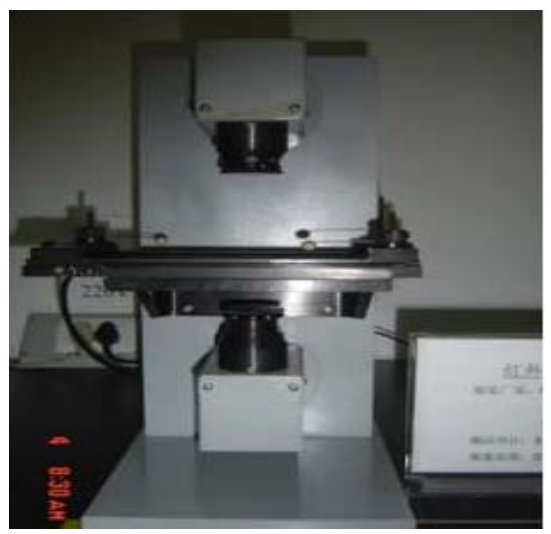

图 2 红外条纹测试仪

Fig.2 Infrared striae tester

\section{3 结果与讨论}

\section{1 条纹严重程度分析}

选择条纹程度由轻微到严重程度不同（其中试样

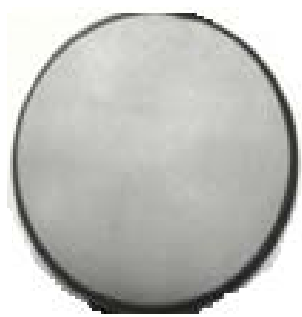

(a) 试样 A (a) Sample A

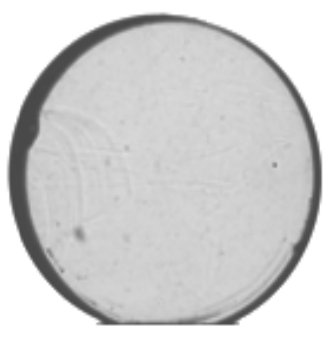

(b) 试样 B (b) Sample B

$\mathrm{A}$ 均匀无明显条纹; 试样 B 内部少量线状、颜色较浅 条纹; 试样 $\mathrm{C}$ 是细而淡的条纹，整个平面的条纹相对 对称; 试样 D 为沟壑状乱条纹) 有代表性的 4 块的 $\mathrm{As}_{40} \mathrm{Se}_{60}$ 红外光学硫系玻璃产品, 用根据以上测试原 理制造出的测试仪器测试其条纹的严重程度, 其测量 结果如图 3 所示。从图中可以看出, 4 块硫系玻璃样 品表面条纹的严重程度具有很大的差异, 这是因为目 前硫系玻璃的生产是采用摇摆炉单块制造，类似于无 色光学玻璃的浇注方式, 其制造工艺不如无色光学玻 璃漏注工艺稳定, 从而决定了产品可获得性及内部质 量一致性比无色光学玻璃、晶体材料都要差。

灰度值是指色彩的浓淡程度。灰度直方图是指一 幅数字图像中, 对应每一个灰度值统计出具有该灰度 值的像素数。黑白图像中点的颜色深度，范围一般从 $0 \sim 255$, 白色为 255 , 黑色为 0 , 目前的图像分析仪 中视频模数转换的精度一般为 8 bits, 即最大灰度级别 值可达 256。实际使用的图像分析仪中，样品的灰度 值都是相对的, 称为灰度级别（或等级）值, 它的大 小由仪器中的视频模数转换装置的精度所决定。视频 模数转换位数越多, 精度越高, 灰度级别就分得越细, 测量图像的细微变化也就越清晰。而玻璃中存在的条 纹会直接引起灰度值的变化。条纹是光学材料重要的 光学指标, 会影响光学元件的传递函数和分辨率, 在 进行光学系统设计时必须对条纹进行限定。本文针对 不透可见光的红外硫系玻璃条纹的测试和表征进行 研究。

\section{2 条纹度对成像结果的影响}

传递函数（modulation transfer function, MTF）是 一种表征玻璃成像质量的方法 ${ }^{[9]}$, 因此, 为了探究条 纹对成像质量的影响，选取 4 块条纹严重程度不同的 直径 $29 \mathrm{~mm}$ 、厚度 $12 \mathrm{~mm}$ 两大面平行、抛光的 $\mathrm{As}_{40} \mathrm{Se}_{60}$ 硫系玻璃, 对单片试样进行MTF测试, 测试结果如图 4 所示。

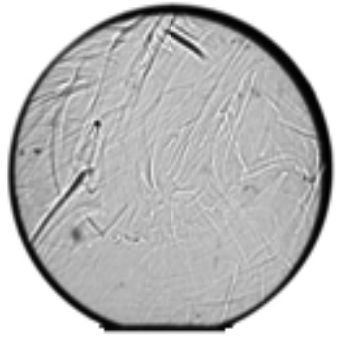

(c) 试样 C (c) Sample C

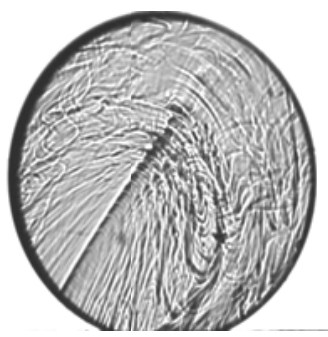

(d) 试样 D (d) Sample D

图 3 红外光学硫系玻璃条纹测量结果

Fig.3 Striae measurement results of infrared chalcogenide optical glass 


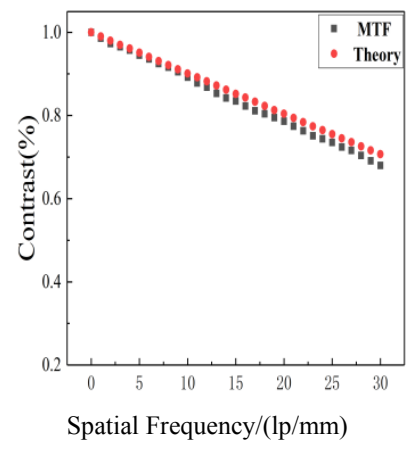

(a) 试样 A 的 MTF 图

(a) MTF graph of sample A

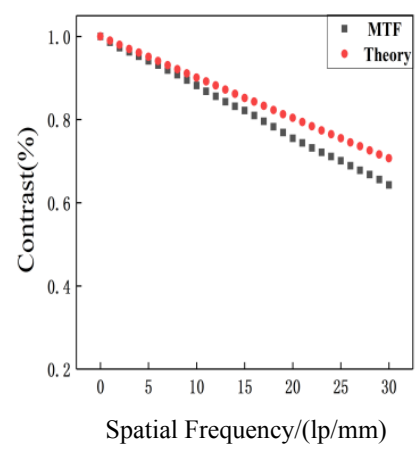

(b)试样 B 的 MTF 图

(b) MTF graph of sample B

图 4 A、B、C、D 试样对应 MTF 函数曲线图

从结果来看, 无明显条纹的试样 A 的 MTF 曲线 与衍射极限接近。内部少量线状、颜色较浅的试样 B 的 MTF 曲线与衍射极限相对接近; 存在细而淡且相 对对称条纹的试样 C 使低频 MTF 迅速下降, 而高频 MTF 稍平缓。这是由较多的、不明显的、均匀的条纹 产生的噪声造成的; 沟壑状乱条纹缺陷的试样 D产生 了大量的底部噪声, 造成 MTF 明显降低。从图中可 以看出, A、B、C、D 4 块玻璃样品的条纹严重程度 依次越来越大, 相应地在与之对应的 MTF 曲线中 MTF 曲线与理论曲线的差异也越来越大。

红外光学玻璃材料的制备方法采用熔融法。在材 料生产制造中, 由于制造工艺的不完善, 会导致材料 局部位置的折射率呈现剧烈变化的现象。折射率急剧 变化的区域就会形成条纹。条纹的形状、粗细、深浅 及面积都会直接影响到成像系统的传递函数以及分 辨率等。

\section{3 条纹度的表示方法研究}

对于无色光学玻璃来讲, 条纹对光学成像质量的 影响与条纹的大小、形状有关, 与玻璃折射率和条纹 折射率之间的差值大小有关。条纹程度或条纹引起的 波前畸变与条纹的几何面积有关, 而且对于那些不能 准确确定位置的条纹, 样品的厚度也会对条纹度产生 重要的影响。连续熔炼方式生产的光学玻璃中条纹主 要是带状条纹。ISO 10110 第 4 部分 ${ }^{[10]}$ 把拋光光学件 的条纹分成 5 个级别。在 $1 \sim 4$ 级中, 条纹度都大于 $30 \mathrm{~nm}$ 的光程差。1 4 级的区分, 按与光学件整个面 积相比较的条纹面积来分级。条纹度小于 $30 \mathrm{~nm}$ 的规 定为 5 级。MIL-G-174 $\mathrm{B}^{[6]}$ 根据条纹程度对毛坏玻璃 的条纹进行分级, 但没有定量考虑条纹面积对成像质 量的影响。

对于红外玻璃来讲, 由于需求量及系统的限制, 目前生产方式决定了条纹存在形式各异。虽然可以采 用红外干涉仪来测量其光程差的变化从而定量度量

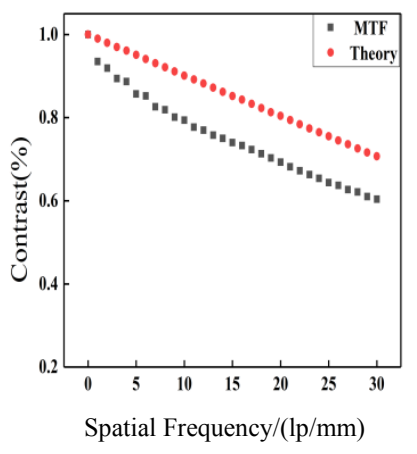

(c)试样 C 的 MTF 图

(c) MTF graph of sample C

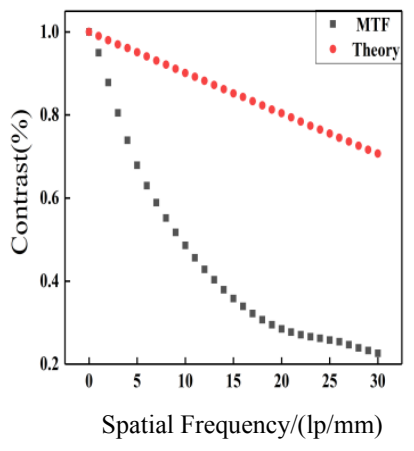

(d) 试样 D 的 MTF 图
Fig.4 MTF graphs of samples A, B, C, D 条纹状况, 但红外干涉仪的价格比较昂贵, 通常只在 有条件的实验室配备, 用以开展系统性研究分析。此 外, 因多数情况下条纹缺陷产生的光程差较大, 用红 外干涉仪无法获得理想的干涉现象, 故无法用红外干 涉仪读取光程差。加之规模化生产时, 需随时对在线 产品抽样检测, 测试批量较大, 所以采用以条纹阴影 图像的灰度和其所占观察图像的面积来快速判定条 纹缺陷严重程度的方法 (简称灰度和面积法), 操作 起来既简便易行，又经济实用。

\subsection{1 条纹灰度的研究}

同一块样品各点的灰度值见图 5。图中不同明暗 处显示的灰度值各不相同, 条纹处的灰度值与无条纹 处的灰度值有相对较大的区别, 灰度值相差较大的区 域呈现的条纹影像更明显。

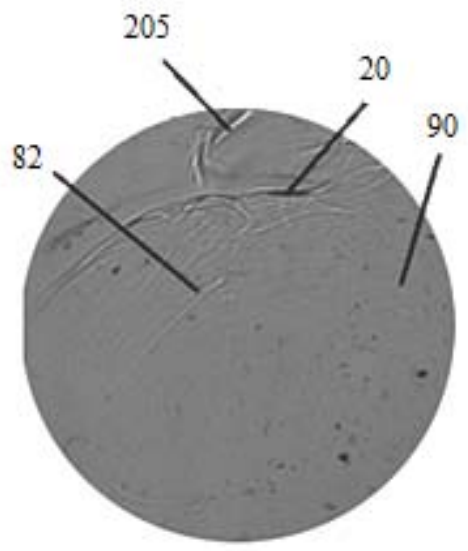

图 5 红外光学硫系玻璃各点灰度值

Fig.5 Gray values of each point for infrared chalcogenide optical glass

因为条纹的产生是局部折射率的急剧变化造成 的, 因此当红外光学硫系玻璃材料中存在条纹时, 条 纹处玻璃的折射率与均匀玻璃的折射率会有明显差 异, 则折射率的变化会导致光线发生偏折, 引起透过 
光线发生不均匀的变化, 在成像探测器靶面上形成阴 影图像。光线偏折会直接导致某处的光强成倍增加或 消失。而透过率的变化可以通过菲涅耳反射定律进行 估算, 在条纹与材料基底界面处透过率的改变量 $\Delta T$ 见式(1):

$$
\Delta T=\left[\frac{1-\left(n_{1}-1\right)^{2}}{1+\left(n_{1}+1\right)^{2}}\right]-\left[\frac{1-\left(n_{2}-1\right)^{2}}{1+\left(n_{2}+1\right)^{2}}\right]
$$

式中: $n_{1}$ 为条纹处玻璃折射率; $n_{2}$ 为玻璃折射率。

假设条纹位置处的折射率与无条纹处玻璃基底 自身折射率的差为 0.005 , 基底折射率为 2.5 , 则在条 纹与材料基底界面处透过率的改变量 $\Delta T$ 为 $7 \times 10^{-4}$ 。 通过公式(1)分析可得, 当被测光学材料的折射率增加 时, 透过率的改变量增加; 当条纹折射率与材料自身 折射率偏差增加时, 透过率的改变量增加; 当条纹的 深度增加时, 透过率的改变量增加。因此成像探测器 分辨出红外光学硫系玻璃中条纹时需要选取适当的 照明强度, 使条纹与被测样品像的强度位于成像探测 器动态范围之内, 无条纹处的灰度值作为标准, 当玻 璃中存在条纹时, 条纹区域透过率的改变将在探测器 上形成条纹的灰度数据。条纹的灰度对成像质量影响 较大, 故选用条纹的灰度值作为评估玻璃内条纹的严 重程度指标之一。当无条纹区域的灰度值作为灰度标 准值时, 以每个条纹区域的平均灰度值与灰度标准值 之差的绝对值来评估玻璃条纹的严重程度。灰度级别 值将随光照条件 (主要指照明光强) 的变化而变化, 欲从灰度级别值获得样品灰度值, 就必须进行灰度值 的绝对标定（根据图像分析仪中样品灰度值的标定原 理）。

一旦光照条件保持不变, 可得到标准灰度样品的 灰度值与其在仪器上测得的灰度级别值之间的关系, 见式(2):

$$
D(j)=A+B \times \lg \left(I_{\mathrm{m}}(j)\right)
$$

因子, 它是与调焦距和镜头光圈有关的量）、 $j$ (为 仪器光学系统透镜组的透光率)、 $I_{\mathrm{i}}$ (入射光强) 有 关的常数, 是 $I_{\mathrm{m}}=1$ 所对应的灰度值, 即确定光照条 件下仪器可测量的最大灰度值; $B$ 应是绝对值为 1 的常数, 它与 1 之间的偏差可视为测量数据的平均 相对误差。

\subsection{2 条纹表征}

一般来讲, 无色光学玻璃的条纹由条纹程度或条 纹引起的波前畸变以及条纹的几何面积来表征。 MIL-G-174 $\mathrm{B}^{[6]}$ 根据条纹程度对毛坏玻璃的条纹进行 分级, 但不考虑条纹面积和样品厚度。将条纹的形状、 粗细与标准样品比较, 把条纹分成 1 4 级 4 个级别。 ISO 10110 第 4 部分 ${ }^{[9]}$ 把抛光光学件的条纹按照条纹 度对光学系统的光程差的影响分成 5 个级别。其技术 标准对比见表 1 。

条纹对光学成像质量的影响与条纹的粗细、形 状有关，与玻璃折射率和条纹折射率之间的差值大 小有关。玻璃折射率和条纹折射率之间的差值产生 波前畸变, 波前畸变的大小与通光方向上的条纹长 度成正比。使用投影法可以在观测方向观测到这种 畸变。在投影屏上, 可以看到条纹的投影。条纹以 灰色的实线出现, 条纹颜色越深, 波前畸变越大。 这种明暗度常常用来表示波前畸变。因此, 可以用 波前畸变和条纹面积来表示其条纹度。而条纹的可 见性与观测方向有关系。条纹度与观测方向的条纹 长度有关系。在条纹的厚度方向观测是看不到条纹 的, 在这个方向使用, 对光学应用也没有任何影响。 而作为硫系红外光学玻璃元件只有通光面方向上的 条纹对于成像质量有影响。

按照以上方法选取同一块硫系玻璃样品、测试同 一区域, 取不同灰度值范围时测试条纹面积百分比及 其条纹灰度, 得出条纹度的测试结果见表 2 。

式中: $A$ 是与 $K$ (反射光或透射光进入镜头的立体角

表 1 MIL 和 ISO 条纹标准与 SCHOTT 技术标准比较

Table 1 Comparison of striae standards among MIL, ISO and SCHOTT

\begin{tabular}{lll}
\hline \multicolumn{1}{c}{ Standard } & \multicolumn{1}{c}{ MIL-G-174B } & \multicolumn{1}{c}{ ISO 10110 } \\
\hline Scope of application & \multicolumn{1}{c}{ Blank glass } & Polished glass \\
\hline Characterization method & Striae(take no account of sample thickness) & Striae area(striae intensity) \\
\hline & Level 4: (no quantitative index) & $\geq 30 \mathrm{~nm}$ \\
Level 3 (no quantitative index) & Level 1: $\leq 10 \% ;$ Level 2: $\leq 5 \%$ \\
\cline { 2 - 3 } Striae level & Level 2: (no quantitative index) & $<30 \mathrm{~nm}$ \\
& Level 1: (no quantitative index) & Level 5: very low striae \\
\cline { 2 - 3 } & &
\end{tabular}


从表 2 可以看出, 取不同的灰度范围值, 条纹面 积及条纹深度的测试结果不同。因此针对不同等级的 产品结合产品的传递函数, 设定合适的灰度值范围, 条纹度的数值为条纹的分级奠定坚实的技术基础。

现选择上述硫系光学玻璃样品按照灰度范围 20
测试条纹度。测试数据见表 3 。

从上述数据可以看出, 采用本文表征方法的条纹 度的数据大小与传递函数的效果相符, 因此, 本文提 出的条纹度指标可以表征红外硫系玻璃产品的成像 质量。

表 2 同一样品不同灰度范围条纹度结果 Table 2 Striae results of different gray levels for the same sample

\begin{tabular}{|c|c|c|c|c|c|}
\hline No. & Setting grey range & Striae area ratio $/ \%$ & & iae grey(standard deviation) & Striae \\
\hline 1 & 5 & $56.91 \%$ & & 26.70 & 15.2 \\
\hline 2 & 10 & $32.15 \%$ & & 34.85 & 11.2 \\
\hline 3 & 15 & $20.16 \%$ & & 42.90 & 8.6 \\
\hline 4 & 20 & $14.22 \%$ & & 49.75 & 7.1 \\
\hline 5 & 30 & $8.72 \%$ & & 60.38 & 5.3 \\
\hline 6 & 50 & $4.32 \%$ & & 76.00 & 3.3 \\
\hline & 表 3 & 条纹度的测试结果 & Table 3 & Test results of striae & \multirow[b]{2}{*}{ Striae } \\
\hline sample & \multicolumn{2}{|c|}{ Striae area ratio $/ \%$} & \multicolumn{2}{|c|}{ Striae grey(standard deviation) } & \\
\hline A & \multicolumn{2}{|c|}{1.12} & \multicolumn{2}{|r|}{15.32} & 0.17 \\
\hline $\mathrm{B}$ & \multicolumn{2}{|c|}{3.53} & \multicolumn{2}{|r|}{28.65} & 1.01 \\
\hline $\mathrm{C}$ & \multicolumn{2}{|c|}{20.68} & \multicolumn{2}{|r|}{35.87} & 7.41 \\
\hline $\mathrm{D}$ & \multicolumn{2}{|c|}{68.34} & \multicolumn{2}{|r|}{65.38} & 44.68 \\
\hline
\end{tabular}

\section{4 结论}

1）本原理采用普通均匀漫射光源照明可获得更 好的测试准确度, 相比 (GB7962.7)、(MIL-G-174B), 本条纹度测试标准实用性更强, 更为先进合理, 可用 于不透可见光的红外硫系光学玻璃条纹度的测试, 也 可用于无色光学玻璃条纹度的测试;

2）通过该设备根据观察到的图像，采用条纹区 域面积和灰度两个标准可以表征玻璃内部的质量状 况, 对光学系统的设计具有重要参考价值。

3）针对红外光学硫系玻璃生产的实际情况, 采 用条纹区域总面积占测试区域总面积的百分比与条 纹灰度的乘积的技术指标来表征红外光学硫系玻璃 的条纹度具有创新性。

\section{参考文献:}

[1] Bacchus JM. Using new optical materials and DOE in low-cost lenses for uncooled IR cameras[C]//Proceedings of SPIE, 2004, 5249: 425-432.

[2] Cogburn G, Symmons A, Mertus L, et al. Molding aspheric lenses for low-cost production versus diamond turned lenses[C]//Proceedings of SPIE, 2010, 7660(20): 1-6.

[3] Kulakova N A, Nasyrov A R, NesmeIova I M. Current trends in creating optical systems for the IR region[J]. Journal of Optical Technology, 2010, 77(5): 324-330.

[4] Ray Hilton. Chalcogenide Glasses for Infrared optics[M]. New York: McGraw Hill, 2010: 131-148.
[5] 杨有金, 卿尚平. 无色光学玻璃测试方法 条纹度检测方法: GB/T 7962.7-1987 [S]. 国家机械工业委员会, 1987-05.

YANG Youjin, QING Shangping. Colorless optical glass test methods Examination method for extent of stria: GB/T 7962.7-1987[S]. National Machinery Industry Committee, 1987-05.

[6] MILITARY SPECIFICATION: GLASS, OPTICAL: MIL-G-174 B[S]. 2004-07-28

[7] 李锡善, 蒋安民, 夏青生. 关于测量光学材料均匀性方法的讨论 [J]. 硅酸盐学报, 1978, 6(3): 149-165.

LI Xishan, JIANG Anmin, XIA Qingsheng. Discussion on the method of measuring the uniformity of optical materials[J]. Journal of the Chinese Ceramic Society, 1978, 6(3): 149-165.

[8] 刘旭, 任寰, 于德强, 等. 干涉法测量光学材料光学非均匀性[J]. 激光 技术, 2011(2): 189-192.

LIU Xu, REN Huan, YU Deqiang, et al. Measurement method for inhomogeneity of optical material by means of interferometry[J]. Laser Technology, 2011(2): 189-192.

[9] 宋宝安, 王乔方, 张莹昭, 等. 红外硒基硫系玻璃光学非均匀性及影 响因素分析 [J]. 红外与激光工程, 2012, 41(8): 1985-1989.

SONG Baoan, WANG Qiaofang, ZHANG Yingzhao, et al. Optical inhomogeneity of IR Se-based chalcogenide glasses and influencing factor analysis[J]. Infrared and Laser Engineering, 2012, 41(8): 1985-1989.

[10] HIS. ISO 10110-part 4, Optics and optical instruments preparation of drawings for optical elements and systems; Material imperfections -inhomogeneity and striae: ISO 10110-5 [S]. 1997. 